PROCEEDINGS OF THE

AMERICAN MATHEMATICAL SOCIETY

Volume 126, Number 9, September 1998, Pages 2699-2703

S 0002-9939(98)04334-2

\title{
MERGELYAN PAIRS FOR HARMONIC FUNCTIONS
}

\author{
STEPHEN J. GARDINER
}

(Communicated by Albert Baernstein II)

\begin{abstract}
Let $\Omega \subseteq \mathbb{R}^{n}$ be open and $E \subseteq \Omega$ be a bounded set which is closed relative to $\Omega$. We characterize those pairs $(\Omega, E)$ such that, for each harmonic function $h$ on $\Omega$ which is uniformly continuous on $E$, there is a sequence of harmonic polynomials which converges to $h$ uniformly on $E$. As an immediate corollary we obtain a characterization of Mergelyan pairs for harmonic functions.
\end{abstract}

\section{Results}

Let $\Omega$ be an open set in Euclidean space $\mathbb{R}^{n}(n \geq 2)$ and let $E \subseteq \Omega$ be a bounded set which is closed relative to $\Omega$. Also, let $\left.f\right|_{E}$ denote the restriction of a function $f$ to $E$. We call $(\Omega, E)$ a Mergelyan pair for harmonic functions if each harmonic function $h$ on $\Omega$ for which $\left.h\right|_{E}$ is uniformly continuous on $E$ can be uniformly approximated by harmonic polynomials on $K \cup E$ for every compact subset $K$ of $\Omega$. This paper presents a complete characterization of Mergelyan pairs for harmonic functions. The corresponding problem for holomorphic functions was solved by Stray [12] in the particular case where $\Omega$ is the unit disc, and then by Brown and Shields [2, p. 79 and Theorem 3] for general plane domains.

We will need some notation. If $K$ is a compact subset of $\mathbb{R}^{n}$, then $K^{\wedge}$ denotes the union of $K$ with the bounded (connected) components of $\mathbb{R}^{n} \backslash K$. Let $\mathcal{A}$ denote the Alexandroff (ideal) point for $\Omega$. If $V$ is a connected open subset of $\Omega$ and there is a continuous function $p:[0,+\infty) \rightarrow V$ such that $p(t) \rightarrow \mathcal{A}$ as $t \rightarrow+\infty$, then we say that $\mathcal{A}$ is accessible from $V$. We define $E^{\sim}$ to be the union of $E$ with the connected components of $\Omega \backslash E$ from which $\mathcal{A}$ is not accessible. Note that the definition of $E^{\sim}$ involves $\Omega$, whereas the definition of $K^{\wedge}$ does not. Also, the notation $\bar{E}^{\wedge}$ means $(\bar{E})^{\wedge}$. We refer to Doob $[6,1 . \mathrm{XI}]$ for an account of thin sets.

Theorem 1. Let $\Omega$ be an open set in $\mathbb{R}^{n}$ and $E \subseteq \Omega$ be a bounded set which is closed relative to $\Omega$. The following are equivalent:

(a) each harmonic function $h$ on $\Omega$ for which $\left.h\right|_{E}$ is uniformly continuous on $E$ can be uniformly approximated on $E$ by harmonic polynomials;

(b) $\mathbb{R}^{n} \backslash \bar{E}^{\wedge}$ and $\mathbb{R}^{n} \backslash E^{\sim}$ are thin at the same points of $\bar{E}$.

Corollary 1. Let $\Omega$ be an open set in $\mathbb{R}^{n}$ and $E \subseteq \Omega$ be a bounded set which is closed relative to $\Omega$. The following are equivalent:

(a) $(\Omega, E)$ is a Mergelyan pair for harmonic functions;

Received by the editors October 21, 1996 and, in revised form, February 3, 1997. 1991 Mathematics Subject Classification. Primary 31B05; Secondary 41A28.

(C)1998 American Mathematical Society 
(b) for each compact subset $K$ of $\Omega$, the sets $\mathbb{R}^{n} \backslash(\bar{E} \cup K)^{\wedge}$ and $\mathbb{R}^{n} \backslash(E \cup K)^{\sim}$ are thin at the same points of $\bar{E} \cup K$.

Corollary 1 is an immediate consequence of Theorem 1 since $\overline{E \cup K}=\bar{E} \cup K$ for each compact $K \subseteq \Omega$. To see that conditions (b) of Corollary 1 and Theorem 1 are distinct, let $B_{1}$ and $B_{2}$ be open balls such that $B_{1}$ is internally tangent to $B_{2}$ at precisely one point, let $\Omega=B_{2}$ and $E=\Omega \cap \partial B_{1}$. Then $\overline{E^{\wedge}}=\overline{B_{1}}$ and $E^{\sim}=E$, so condition (b) of Theorem 1 holds. However, if $K=\{x\}$, where $x$ is the centre of $B_{1}$, then $(E \cup K)^{\sim}=E \cup K$ and $x$ is in the interior of $(\bar{E} \cup K)^{\widehat{ }}$, so condition (b) of Corollary 1 fails. Similar considerations show that condition (b) of Corollary 1 implies that $\bar{E}^{\wedge} \cap \Omega=E^{\sim}$, and even that $(\bar{E} \cup K)^{\wedge} \cap \Omega=(E \cup K)^{\sim}$ for every compact $K \subseteq \Omega$.

We note from Brown and Shields [2, Theorem 3] that a Mergelyan pair for holomorphic functions must satisfy $\overline{E^{\sim}}=\bar{E}^{\wedge}$. To see that this is not the case for harmonic functions, let $\Omega=B_{2} \backslash \overline{B_{1}}$, where $B_{1}$ and $B_{2}$ are open balls as above. Further, let $E$ be a countable subset of $\Omega$ whose set of accumulation points is $\partial B_{1}$. Then $(\Omega, E)$ satisfies condition (b) of Corollary 1, but $\overline{E^{\sim}}=E \cup \partial B_{1} \neq E \cup \overline{B_{1}}=\bar{E}^{\wedge}$.

Let $B(x, r)$ denote the open ball in $\mathbb{R}^{n}$ with centre $x$ and radius $r$. For any set $F$ in $\mathbb{R}^{n}$ we define

$$
F^{\vee}=F \cup\left\{x \in \mathbb{R}^{n}: B(x, r) \backslash F \text { is polar for some } r>0\right\} .
$$

Clearly $F^{\vee} \backslash F$ is a polar set. We will show that, when $n=2$, condition (b) of Theorem 1 simplifies to the condition that $\partial\left(\bar{E}^{\wedge}\right)=\partial\left(\left(E^{\sim}\right)^{\vee}\right)$. Thus we have the following.

Corollary 2. Let $\Omega$ be an open set in $\mathbb{R}^{2}$ and $E \subseteq \Omega$ be a bounded set which is closed relative to $\Omega$. The following are equivalent:

(a) $(\Omega, E)$ is a Mergelyan pair for harmonic functions;

(b) $\partial\left((\bar{E} \cup K)^{\wedge}\right)=\partial\left(\left((E \cup K)^{\sim}\right)^{\vee}\right)$ for each compact subset $K$ of $\Omega$.

Corollary 2 extends recent work of Bonilla, Perez-Gonzalez and Trujillo-Gonzalez [1, Theorem 3.3 and Correction] which characterizes Mergelyan pairs for harmonic functions in the plane in the special case where $\Omega$ is a bounded open set satisfying $\partial \Omega=\partial\left(\bar{\Omega}^{\wedge}\right)$.

\section{ProOFs}

2.1. Suppose that condition (b) of Theorem 1 holds and let $h$ be a harmonic function on $\Omega$ such that $\left.h\right|_{E}$ is uniformly continuous on $E$. Then $h$ can be extended to $\Omega \cup \bar{E}$ in such a way that the restriction of $h$ to $\bar{E}$ is continuous.

We claim that the restriction of $h$ to $\overline{E^{\sim}}$ is continuous. To see this, let $W=$ $E^{\sim} \backslash E$. Then each point $y$ of $\partial W \cap \partial \Omega$ is regular for the Dirichlet problem on $W$; for otherwise, $\mathbb{R}^{n} \backslash W$ would be thin at $y$, and a result of Deny [5] then yields the contradictory conclusion that there are (many) paths emanating from $y$ which lie initially in $W$. Also, it is clear that $\partial W \subseteq \bar{E}$. Let $u=H_{h}^{W}-h$, where $H_{h}^{W}$ denotes the Perron-Wiener-Brelot solution of the Dirichlet problem on $W$ with boundary function $h$. Then $u(x) \rightarrow 0$ as $x \rightarrow y$ for each $y \in \partial W \cap \Omega$ which is regular for $W$, and $\lim \sup _{x \rightarrow y}|u(x)|<+\infty$ for each $y$ in the polar set of irregular boundary points of $W$. Let $w$ be a positive superharmonic function on an open set containing 
$\overline{E^{\sim}}$ such that $w=+\infty$ on this polar set. If $\delta>0$, then

$$
\limsup _{x \rightarrow y, x \in W}\{|u(x)|-\delta w(x)\} \leq 0 \quad(y \in \partial W \cap \Omega) .
$$

Since $\mathcal{A}$ is not accessible from any component of $W$, it follows that $|u|-\delta w \leq 0$ on $W$ (see [3], for example), and since $\delta$ can be arbitrarily small, we can conclude that $u \equiv 0$. Thus, by the regularity of points of $\partial W \cap \partial \Omega$ for $W$, the restriction of $h$ to $\overline{E^{\sim}}$ is continuous.

Let $F=\overline{E^{\sim}}$ and let $A$ denote the fine interior of $F$. Then $h$ is finely harmonic on $A \cap \Omega$ (see [7] for an account of finely harmonic functions). Noting that $F \subseteq \bar{E}^{\wedge}$, we see from condition (b) that

$$
\begin{aligned}
A \backslash \Omega & =\left\{x \in \bar{E} \cap \partial \Omega: \mathbb{R}^{n} \backslash F \text { is thin at } x\right\} \\
& \subseteq\left\{x \in \bar{E} \cap \partial \Omega: \mathbb{R}^{n} \backslash \bar{E}^{\wedge} \text { is thin at } x\right\} \\
& =\left\{x \in \bar{E} \cap \partial \Omega: \mathbb{R}^{n} \backslash E^{\sim} \text { is thin at } x\right\} \\
& \subseteq\left\{x \in \partial \Omega: \mathbb{R}^{n} \backslash \Omega \text { is thin at } x\right\},
\end{aligned}
$$

and this latter set is polar. Since polar sets are removable for bounded finely harmonic functions (see [7, Theorem 9.15]), $h$ is finely harmonic on $A$.

Let $\varepsilon>0$. In view of the properties of $h$ established in the preceding two paragraphs, we can apply a result of Debiard and Gaveau [4] to see that there is a harmonic function $\nu$ on a neighbourhood of $F$ such that $|h-\nu|<\varepsilon / 2$ on $F$. Since $E^{\sim} \subseteq F \subseteq F^{\wedge}=\bar{E}^{\wedge}$, we see from condition (b) that $\mathbb{R}^{n} \backslash F^{\wedge}$ and $\mathbb{R}^{n} \backslash F$ are thin at the same points of $\bar{E}$, and hence at the same points of $F$. By $[8$, Theorem 1.10], there is a harmonic function $h_{0}$ on $\mathbb{R}^{n}$ such that $\left|\nu-h_{0}\right|<\varepsilon / 2$ on $F$, and hence $\left|h-h_{0}\right|<\varepsilon$ on $\bar{E}$. By suitably truncating the expansion of $h_{0}$ in terms of homogeneous harmonic polynomials, we obtain condition (a) of Theorem 1.

2.2. Conversely, suppose that condition (a) of Theorem 1 holds. Since $E^{\sim} \subseteq \bar{E}^{\wedge}$, it is enough to show that, if $\mathbb{R}^{n} \backslash E^{\sim}$ is non-thin at a point $y$ in $\bar{E}$, then so also is $\mathbb{R}^{n} \backslash \bar{E}^{\curlywedge}$. If $n=2$, then let $\omega$ denote an open disc which contains $\bar{E}$; otherwise let $\omega=\mathbb{R}^{n}$. Thus, in either case, $\omega$ possesses a Green function. Let $u^{\#}$ denote a continuous potential on $\omega$ which determines thinness (see [6, 1.XI.10]), and let $\widehat{R}_{u}^{A}$ denote the regularized reduced function (balayage) of a positive superharmonic function $u$ relative to a set $A$ in $\omega$. Also, let $B$ be an open ball such that $\bar{E} \subset B$ and $\bar{B} \subset \omega$.

Suppose that $\mathbb{R}^{n} \backslash E^{\sim}$ is non-thin at a fixed point $y$ of $\bar{E}$ and let $\varepsilon>0$. For each $m$ in $\mathbb{N}$ let

$$
A_{m}=B \backslash\left[B(y, 1 / m) \cup\left\{x \in \Omega: \operatorname{dist}\left(x, E^{\sim}\right)<1 / m\right\}\right] .
$$

Since $A_{m} \uparrow B \backslash\left(E^{\sim} \cup\{y\}\right)$ and $\mathbb{R}^{n} \backslash E^{\sim}$ is non-thin at $y$, we see that

$$
\widehat{R}_{u^{\#}}^{A_{m}}(y) \uparrow \widehat{R}_{u^{\#}}^{B \backslash E^{\sim}}(y)=u^{\#}(y) \quad(m \rightarrow \infty) .
$$

We choose $k$ large enough so that

$$
\widehat{R}_{u^{\#}}^{A_{k}}(y) \geq u^{\#}(y)-\varepsilon .
$$

The measure $\mu$ associated with the potential $\widehat{R}_{u^{\#}}^{A_{k}}$ has support $\bar{A}_{k}$. It follows from a theorem of Choquet (see [11, Theorem 6.21]) and the fact that $y \notin \bar{A}_{k}$, that we 
can restrict $\mu$ to a smaller set in such a way that the corresponding potential $u$ is continuous on $\omega$ and satisfies

$$
u(y)>u^{\#}(y)-2 \varepsilon .
$$

We now appeal to a recent result of the author [10, Theorem 2(b)]. This asserts that, if $u$ is uniformly continuous on $E^{\sim}$ and finely harmonic on the fine interior of $E^{\sim}$, then $u$ can be uniformly approximated on $E^{\sim}$ by harmonic functions on $\Omega$ whose restrictions to $E^{\sim}$ are uniformly continuous. The hypotheses of this result are certainly satisfied in the case of the function $u$ constructed in the previous paragraph, since $u$ is continuous on $\omega$ and harmonic on the open set $\left\{x \in \Omega\right.$ : $\left.\operatorname{dist}\left(x, E^{\sim}\right)<1 / k\right\}$, which contains $E^{\sim}$. Hence there is a harmonic function $h$ on $\Omega$ such that $\left.h\right|_{E^{\sim}}$ is uniformly continuous on $E^{\sim}$ and such that

$$
|u(x)-h(x)|<\varepsilon \quad\left(x \in E^{\sim}\right) .
$$

Let $L=\bar{E}^{\curlywedge}$. We next apply condition (a) to obtain a harmonic polynomial $h_{0}$ such that $\left|h-h_{0}\right|<\varepsilon$ on $E$. Thus, in view of $(2),\left|u-h_{0}\right| \leq 2 \varepsilon$ on $\bar{E}$, which contains $\partial L$. If we define

$$
V_{m}=\{x \in \omega: \operatorname{dist}(x, L)<1 / m\} \quad(m \in \mathbb{N}),
$$

then for large values of $m$,

$$
\left|H_{u}^{V_{m}}-h_{0}\right|=\left|H_{u-h_{0}}^{V_{m}}\right|<3 \varepsilon \quad \text { on } \bar{E} .
$$

Since $H_{u}^{V_{m}}=\widehat{R}_{u}^{\omega \backslash V_{m}}$ on $V_{m}$, we see that

$$
\left|\widehat{R}_{u}^{\omega \backslash V_{m}}-u\right| \leq\left|\widehat{R}_{u}^{\omega \backslash V_{m}}-h_{0}\right|+\left|h_{0}-u\right|<5 \varepsilon \quad \text { on } \bar{E} .
$$

From (3), (1) and the fact that $u^{\#} \geq u$ we obtain

$$
\widehat{R}_{u \#}^{\omega \backslash L}(y) \geq \widehat{R}_{u}^{\omega \backslash L}(y) \geq u(y)-5 \varepsilon>u^{\#}(y)-7 \varepsilon .
$$

Since $\varepsilon$ can be arbitrarily small, we see that

$$
\widehat{R}_{u \#}^{\omega \backslash L}(y)=u^{\#}(y) .
$$

It follows that $\omega \backslash L$, and hence $\mathbb{R}^{n} \backslash L$, is non-thin at $y$, as required.

2.3. In order to prove Corollary 2 , it is enough to show that, when $n=2$, condition (b) of Theorem 1 can be replaced by:

$\left(\mathrm{b}^{\prime}\right) \partial\left(\bar{E}^{\wedge}\right)=\partial\left(\left(E^{\sim}\right)^{\vee}\right)$.

First suppose that condition ( $\left.\mathrm{b}^{\prime}\right)$ holds and suppose further that $\mathbb{R}^{2} \backslash \bar{E}^{\curlywedge}$ is thin at a point $y$ of $\bar{E}$. It follows that there are (many) circles centred at $y$ which are contained in $\bar{E}^{\curlywedge}$. Hence $y$ lies in the interior of $\bar{E}^{\curlywedge}$. By condition $\left(\mathrm{b}^{\prime}\right), y \notin \partial\left(\left(E^{\sim}\right)^{\vee}\right)$. Clearly $y \in \overline{\left(E^{\sim}\right)^{\vee}}$, so $\mathbb{R}^{2} \backslash\left(E^{\sim}\right)^{\vee}$ is thin at $y$. Since $\left(E^{\sim}\right)^{\vee} \backslash E^{\sim}$ is polar, it follows that $\mathbb{R}^{2} \backslash E^{\sim}$ is thin at $y$. Thus condition (b) of Theorem 1 holds.

Conversely, suppose that condition $\left(\mathrm{b}^{\prime}\right)$ fails to hold. We will show that condition (a) of Theorem 1 then fails. Let $F=\overline{E^{\sim}}$ and let $\omega$ be an open disc that contains $F$. Since $F^{\wedge}=\bar{E}^{\curlywedge}$, it is clear that $\partial\left(\bar{E}^{\wedge}\right) \subseteq \partial F \subseteq \partial\left(\left(E^{\sim}\right)^{\vee}\right)$. Thus there exists a point $y$ in $\partial\left(\left(E^{\sim}\right)^{\vee}\right) \backslash \partial\left(\bar{E}^{\wedge}\right)$. We note that $y \in F \subseteq \bar{E}^{\wedge}$, so $y \in\left(\bar{E}^{\wedge}\right)^{0}$. Let $r>0$ be such that $B(y, 2 r) \subseteq \bar{E}^{\curlywedge}$. Since $y \in \partial\left(\left(E^{\sim}\right)^{\vee}\right)$, it follows that $B(y, r) \backslash E^{\sim}$ is non-polar. Thus we can choose a non-zero measure on $B(y, r) \backslash E^{\sim}$ such that the 
corresponding potential $u$ on $\omega$ is continuous (in addition to being harmonic on $\left.\left(E^{\sim}\right)^{0}\right)$ and satisfies

$$
u(y)>\sup \left\{u(x): x \in \partial\left(\bar{E}^{\wedge}\right)\right\} .
$$

By $[9$, Corollary 1] (or [10, Corollary 2]) there is a function $h$ which is harmonic on $\Omega$ such that $\left.h\right|_{E}$ is uniformly continuous on $E$, and such that

$$
h(y)>\sup \left\{h(x): x \in \partial\left(\bar{E}^{\wedge}\right)\right\} .
$$

It is now clear that condition (a) of Theorem 1 fails, for otherwise (4) would lead us to contradict the maximum principle for harmonic functions in view of the fact that $y \in\left(\bar{E}^{\wedge}\right)^{0}$.

The proof of Corollary 2 is now complete.

\section{REFERENCES}

[1] A. Bonilla, F. Pérez-González and R. Trujillo-González, Mergelyan sets for certain classes of harmonic functions, Complex Variables 31 (1996), 9-18; Correction, to appear. CMP 97:05

[2] L. Brown and A. L. Shields, Approximation by analytic functions uniformly continuous on a set, Duke Math. J. 42 (1975), 71-81. MR 51:3458

[3] Chen Huaihui and P. M. Gauthier, A maximum principle for subharmonic and plurisubharmonic functions, Canad. J. Math. 35 (1992), 34-39. MR 93a:31013

[4] A. Debiard and B. Gaveau, Potentiel fin et algèbres de fonctions analytiques I, J. Funct. Anal. 16 (1974), 289-304. MR 52:1326

[5] J. Deny, Un théorème sur les ensembles effilés, Ann. Univ. Grenoble Sect. Sci. Math. Phys. 23 (1948), 139-142. MR 9:509a

[6] J. L. Doob, Classical potential theory and its probabilistic counterpart, Springer, New York, 1983. MR 85k:31001

[7] B. Fuglede, Finely harmonic functions, Lecture Notes in Math. 289, Springer, Berlin, 1972. MR 56:8883

[8] S. J. Gardiner, Harmonic approximation, London Math. Soc. Lecture Notes 221, Cambridge University Press, 1995. MR 96j:31001

[9] S. J. Gardiner, Uniform harmonic approximation with continuous extension to the boundary, J. Analyse Math. 68 (1996), 95-106. CMP 96:16

[10] S. J. Gardiner, Decomposition of approximable harmonic functions, Math. Ann. 308 (1997), 175-185. CMP 97:11

[11] L. L. Helms, Introduction to potential theory, Krieger, New York, 1975. MR 57:659

[12] A. Stray, Characterization of Mergelyan sets, Proc. Amer. Math. Soc. 44 (1974), 347-352. MR 50:13543

Department of Mathematics, University College Dublin, Dublin 4, Ireland

E-mail address: stephen.gardiner@ucd.ie 